\title{
Marfan syndrome and symptomatic dural ectasia: A case report and literature review
}

\author{
Si Nae Eom, Dong Chan Kim, Kwang Nam Kim, and Sung Hye Kim* \\ Department of Pediatrics, Hallym University Sacred Heart Hospital, Anyang, Korea
}

Dural ectasia refers to the widening or ballooning of the dural sac surrounding the spinal cord. It can affect any plane of the spinal canal, but occurs primarily in the lumbosacral region. Dural ectasia is present in 63-92\% patients who have Marfan syndrome, and is related to Ehlers-Danlos syndrome, neurofibromatosis type I, and ankylosing spondylitis. The most common symptoms are low back pain, headache, weakness, numbness above and below the affected limb, and occasional rectal and genital pain. However, in most patients, dural ectasia is usually asymptomatic. We report the case of a 5-year-old boy who presented with a severe headache who had been diagnosed with Marfan syndrome. During the evaluation, magnetic resonance imaging of the lumbar and sacral spine revealed dural ectasia. To our knowledge, this is the first report on Marfan syndrome with symptomatic dural ectasia in Korea. We concluded that dural ectasia should be suspected in patients diagnosed with Marfan syndrome who have a severe headache.

Key words: Marfan syndrome, Headache.

\section{Introduction}

Marfan syndrome is an autosomal dominant genetic disorder caused by a defect in connective tissue that supports the body structures [1]. It affects the ocular, cardiovascular, pulmonary, nervous, and musculoskeletal systems [2]. A diagnosis of Marfan syndrome is based on well-defined clinical criteria (the revised Ghent diagnostic criteria) [3].

Dural ectasia involves enlargement of the dural sac that surrounds the central nervous system [4]. The most common symptoms are low back pain, headache, weakness, numbness above and below the affected limb, and occasional rectal and genital pain; however, in most patients, dural ectasia is usually asymptomatic [5]. The exact prevalence in pediatric patients is not known. In one report, the prevalence of dural ectasia was 90.3\% in pediatric patients with Marfan syndrome [6]. Dural ectasia is one factor in the systemic scoring system for clinical diagnosis of Marfan syndrome [3]. Despite its relatively high prevalence, dural ectasia has rarely been reported in children with Marfan syndrome in Korea. Recently, we treated a 5-yearold boy with a severe headache who had been diagnosed with Marfan syndrome based on clinical findings. During the evaluation for the headache, dural ectasia was revealed on magnetic resonance imaging (MRI) of the spine.

Received: 19 November 2014, Revised: 13 December 2014, Accepted: 13 December 2014, Published: 31 December 2014

${ }^{*}$ Corresponding author: Sung Hye Kim, M.D.

Department of Pediatrics, Hallym University Sacred Heart Hospital, 22 Gwanpyeong-ro 170beon-gil, Dongan-gu, Anyang 431-796, Korea.

Tel: +82-31-380-1685, Fax: +82-31-380-1900, E-mail: rohetkim@yahoo.co.kr

Conflict of interest: We declare that we do not have any conflicts of interests.

(c) This is an open-access article distributed under the terms of the Creative Commons Attribution Non-Commercial License (http://creativecommons.org/licenses/by-nc/3.0/) which permits unrestricted non-commercial use, distribution, and reproduction in any medium, provided the original work is properly cited.

(c) Copyright 2014 by the Korean Society of Medical Genetics 


\section{Case}

A 5-year-old boy visited the pediatric outpatient clinic at Hallym University Sacred Heart Hospital with a severe headache, along with backache and vomiting for 2 days. Two months before, he had been diagnosed with Marfan syndrome based on clinical findings. He was $116.5 \mathrm{~cm}$ tall and weighed $20 \mathrm{~kg}$ at admission. His body mass index was $14.7 \mathrm{~kg} / \mathrm{m}^{2}$. His skeletal system had characteristic morphology, including enophthalmos, arachnodactyly, increased arm/height span, and positive thumb sign. His cardiovascular system had aortic root dilatation (aortic valve $[\mathrm{AV}]$ annulus Z-score 3.08, AV sinus Z-score 5.51) on echocardiography. An ophthalmologic examination revealed mild lens dislocations in both eyes. Based on these findings, he was clinically diagnosed with Marfan syndrome based on the revised Ghent nosology. In addition, genetic testing was performed using FBN1 gene sequencing; however, no FBN1 gene mutation was found.

According to his medical history, he had, on separate occasions, undergone herniorrhaphy for right inguinal hernia and been admitted because of small bowel intussusception 4 years before. There was no history of Marfan syndrome in his family, which included his parents and an elder brother.

On admission, he presented with an acute sickly appearance owing to the severe headache. He had no fever, neck stiffness, Bruzinsky's sign, or Kernig's sign. A chest $X$-ray revealed cardiomegaly and T wave inversion in leads $I I, I I$, and aVF was observed on electrocardiography. After admission, his headache worsened. On computed tomography of the brain, there were no abnormal findings except for the incidental maxillary sinusitis. He had no signs of increased intracranial pressure, such as papilledema, on fundus examination. We performed a spinal tap to exclude the possibility of infection in the central nervous system. A notable finding was the "dry tap" on lumbar puncture, although the needle was inserted appropriately several times of trial. We could get only collect a small amount of cerebrospinal fluid (CSF) and could not accurately measure CSF pressure. The CSF results were all within normal ranges (specific gravity, 1.009; white blood cells, $0 / \mu \mathrm{L}$; red blood cells, $0 / \mu \mathrm{L}$; glucose, $68 \mathrm{mg} /$ $\mathrm{dL}$; and protein $44.5 \mathrm{mg} / \mathrm{dL}$ ). We treated him with analgesics to relieve the headache and antibiotics to treat the incidentally found sinusitis. His headache was relieved when he lay supine but aggravated when he sat up. We considered the headache to be a symptom related to his Marfan syndrome. For evaluation, we conducted an MRI of the spine. The spinal canal was
(A)

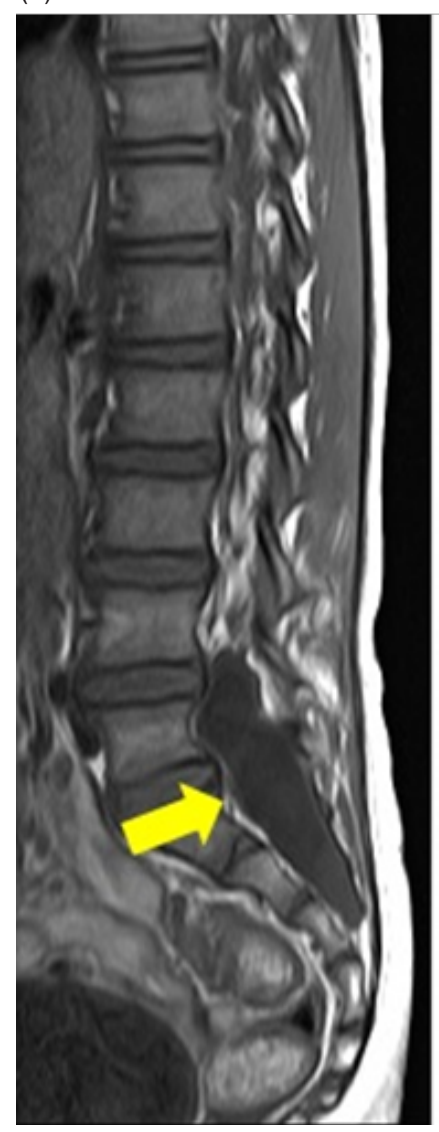

(B)

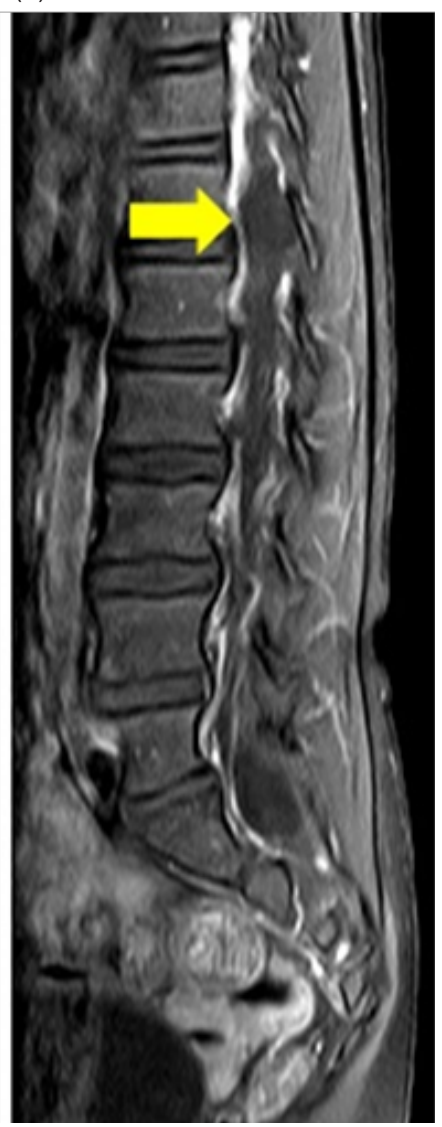

Fig. 1. (A) Expansile dural sac enlargement with expansion of the spinal canal at the L4-S3 level (arrow). Smooth C-shaped scalloping of the posterior vertebral body of $L 5$ is noted. (B) Dural sac enlargement at the T12-L1 level (arrow).

expanded at the L4-S3 and T12-L1 levels with expansile dural sac enlargement (Fig. 1).

Because headache could be provoked by CSF leakage from the dural sac, an epidural blood patch was considered. However, after dural ectasia was diagnosed on MRI, the patient's headache improved, and he could sit up without pain. Therefore, we cared for the patient conservatively with hydration and bed rest. He was discharged from the hospital and thus far has had no recurrent headache.

\section{Discussion}

Dural ectasia was considered a major diagnostic criterion for Marfan syndrome, equal to lens dislocation and aortic root enlargement, in the original Ghent nosology [7]. However, since the criteria were revised in 2010, dural ectasia is no longer a 
major diagnostic criterion but just one element of the systemic scoring system. Therefore, the importance of dural ectasia has become undervalued for the diagnosis of Marfan syndrome [8]. However, pathologic FBN1 gene mutations are not found in all patients who are thought to have Marfan syndrome [9]. Thus, considering its high prevalence, dural ectasia remains a critical diagnostic criterion for early clinical diagnosis of Marfan syndrome [10].

We examined a 5-year-old boy with a severe headache who had been diagnosed with Marfan syndrome and, following an MRI of the spine, was diagnosed with dural ectasia. The confirmation of dural ectasia may contribute to the clinical diagnosis as one element of the systemic scoring system, even though a gene mutation was not confirmed. Marfan syndrome must be diagnosed at an early age so that complications from cardiac manifestations (mitral valve prolapse, heart failure, and aortic dissection) can be prevented in the early stages [6]. Despite the relatively high prevalence, diagnosis of Marfan syndrome is often delayed due to variability in the manifestation of clinical features in children [1]. Therefore, we emphasize the necessity of early screening for dural ectasia in individuals with characteristic features, in order to diagnose Marfan syndrome and start preventive care of complications early in life.

Dural ectasia is usually asymptomatic [5]. However, as in our case, it can trigger headache. This occurs because of excessive CSF accumulation in the lumbosacral region, or CSF leaks owing to underlying structural weakness of the spinal meninges, causing low pressure and postural hypotension [11]. In addition, it is hypothesized that pain occurs from displacement of painsensitive structures in the skull when patients stand up, but this mechanism is not clear [12]. Treatment is usually conservative, with sufficient hydration and bed rest [13]. If symptoms are not relieved, more invasive treatments can be tried, including the epidural blood patch, continuous epidural saline infusion, or an epidural injection of fibrin glue [14]. If symptoms persist, direct surgical repair may be necessary [14]. Based on this case, we concluded that dural ectasia should be suspected in patients with Marfan syndrome who have a severe headache.

\section{References}

1. Tinkle BT, Saal HM; Committee on Genetics. Health supervision for children with Marfan syndrome. Pediatrics 2013;132:e1059-72.

2. Pyeritz RE, McKusick VA. The Marfan syndrome: diagnosis and management. N Engl J Med 1979;300:772-7.

3. Dean JC. Marfan syndrome: clinical diagnosis and management. Eur J Hum Genet 2007;15:724-33.

4. Pyeritz RE, Fishman EK, Bernhardt BA, Siegelman SS. Dural ectasia is a common feature of the Marfan syndrome. Am J Hum Genet 1988;43:726-32.

5. Altman A, Uliel L, Caspi L. Dural ectasia as presenting symptom of Marfan syndrome. Isr Med Assoc J 2008;10:194-5.

6. Veldhoen S, Stark V, Mueller GC, Derlin T, Bley TA, Weil J, et al. Pediatric patients with Marfan syndrome: frequency of dural ectasia and its correlation with common cardiovascular manifestations. Fortschr Röntgenstr 2014;186:61-6.

7. Loeys BL, Dietz HC, Braverman AC, Callewaert BL, De Backer J, Devereux RB, et al. The revised Ghent nosology for the Marfan syndrome. J Med Genet 2010;47:476-85.

8. De Paepe A, Devereux RB, Dietz HC, Hennekam RC, Pyeritz RE. Revised diagnostic criteria for the Marfan syndrome. Am J Med Genet 1996;62:417-26.

9. Attanasio M, Lapini I, Evangelisti L, Lucarini L, Giusti B, Porciani M, et al. FBN1 mutation screening of patients with Marfan syndrome and related disorders: detection of 46 novel FBN1 mutations. Clin Genet 2008;74:39-46.

10. Rose PS, Levy HP, Ahn NU, Sponseller PD, Magyari T, Davis J, et al. A comparison of the Berlin and Ghent nosologies and the influence of dural ectasia in the diagnosis of Marfan syndrome. Genet Med $2000: 2: 278-82$.

11. Schievink WI. Spontaneous spinal cerebrospinal fluid leaks and intracranial hypotension. JAMA 2006;295:2286-96.

12. Rando TA, Fishman RA. Spontaneous intracranial hypotension: report of two cases and review of the literature. Neurology 1992;42:481-7.

13. Schick U, Musahl C, Papke K. Diagnostics and treatment of spontaneous intracranial hypotension. Minim Invasive Neurosurg 2010:53:15-20.

14. Schievink WI, Meyer FB, Atkinson JL, Mokri B. Spontaneous spinal cerebrospinal fluid leaks and intracranial hypotension. J Neurosurg 1996;84:598-605. 